\title{
Loss of Presynaptic Terminal Integrity in the Substantia Nigra in Early Parkinson's Disease
}

\author{
Aline Delva MD, ${ }^{1,2^{*}}$ (D) Donatienne Van Weehaeghe MD, ${ }^{3,4}$ Michel Koole PhD, ${ }^{4}$ Koen Van Laere MD, PhD, ${ }^{3,4}$ and \\ Wim Vandenberghe MD, $\mathrm{PhD}^{1,2}$ \\ ${ }^{1}$ Department of Neurosciences, KU Leuven, Flanders, Belgium \\ ${ }^{2}$ Department of Neurology, University Hospitals Leuven, Flanders, Belgium \\ ${ }^{3}$ Division of Nuclear Medicine, University Hospitals Leuven, Flanders, Belgium \\ ${ }^{4}$ Nuclear Medicine and Molecular Imaging, Department of Imaging and Pathology, KU Leuven, Flanders, Belgium
}

\begin{abstract}
Background: It has been hypothesized that the pathology of Parkinson's disease (PD) primarily affects presynaptic terminals and spreads transsynaptically.

Objectives: The main objective of this study was to assess the magnitude and anatomical extent of presynaptic terminal loss across the brain in early PD. A second objective was to compare loss of presynaptic terminals and cell bodies within the nigrostriatal tract.

Methods: A total of 30 patients with early PD and 20 age- and gender-matched healthy controls underwent positron emission tomography with ${ }^{11} \mathrm{C}-\mathrm{UCB}-\mathrm{J}$, a ligand for the universal presynaptic terminal marker synaptic vesicle protein $2 \mathrm{~A}(\mathrm{SV} 2 \mathrm{~A})$, and with the dopamine transporter ligand ${ }^{18} \mathrm{~F}$-FE-PE2I, as well as a detailed clinical assessment. Volumes of interest were delineated based on individual 3-dimensional T1 magnetic resonance imaging. $\mathrm{BP}_{\mathrm{ND}}$ images were calculated.

Results: Patients with PD showed significant loss of SV2A binding in the substantia nigra only. Loss of dopamine transporter binding in the PD group was much
\end{abstract}

greater in the putamen than in the substantia nigra. We found no correlations between SV2A or dopamine transporter binding and any of the clinical motor or nonmotor scores. Homologous voxel-based analysis in the PD group showed significant correlations between SV2A and dopamine transporter binding in the caudate and substantia nigra.

Conclusions: Presynaptic terminals appear to be the most heavily affected subcellular compartment of nigrostriatal neurons in early PD. Moreover, early PD causes loss of presynaptic terminals that innervate the nigrostriatal neurons. This loss of presynaptic boutons in the substantia nigra may reflect an axonal response to target deprivation or could possibly point to a transsynaptic mode of propagation of the disease process. (C) 2020 International Parkinson and Movement Disorder Society

Key Words: ${ }^{11} \mathrm{C}-U \mathrm{CB}-\mathrm{J}$; dopamine transporter; PET; SV2A; synaptic density

Parkinson's disease (PD) is a leading cause of neurodegeneration and has traditionally been defined *Correspondence to: Dr. Aline Delva, KU Leuven, Herestraat 49, 3000
Leuven, Belgium; E-mail: aline.delva@kuleuven.be

Funding agencies: Supported by KU Leuven ("Opening the Future" and C24/17/063) and UZ Leuven (Fund for Academic Studies and Klinische Onderzoeks- en Opleidingsraad).

Relevant conflicts of interests/financial disclosures: Aline Delva and Donatienne Van Weehaeghe are PhD fellows of the Fund for Scientific Research Flanders. Wim Vandenberghe is a Senior Clinical Investigator of the Fund for Scientific Research Flanders.

Received: 10 April 2020; Revised: 26 May 2020; Accepted: 22 June 2020

Published online 00 Month 2020 in Wiley Online Library (wileyonlinelibrary.com). DOI: 10.1002/mds.28216 by the loss of dopaminergic neurons in the substantia nigra and the presence of intraneuronal $\alpha$-synuclein inclusions (Lewy bodies and Lewy neurites). ${ }^{1}$ However, it is now clear that neuronal loss and Lewy pathology in PD also affect many nondopaminergic cell populations, ${ }^{2,3}$ and several observations suggest that the disease process may spread trans-synaptically between anatomically connected brain regions. ${ }^{4-7}$

Mounting evidence suggests that the pathological process of PD may be initiated presynaptically. In the healthy brain, $\alpha$-synuclein is highly enriched in presynaptic terminals. ${ }^{8}$ In brains of patients with PD or dementia with Lewy bodies, at least $90 \%$ of aggregated $\alpha$-synuclein is located presynaptically and not in 
somatic Lewy bodies. ${ }^{9,10}$ In cell culture models of PD, $\alpha$-synuclein misfolding begins in presynaptic terminals, where it induces loss of synaptic vesicle-associated proteins and synaptic dysfunction and propagates from there to the cell bodies. ${ }^{11}$ Postmortem studies of patients with PD and incidental Lewy body disease also indicate that $\alpha$-synuclein aggregation and neurodegeneration may begin in distal axons and then spread to neuronal somata. ${ }^{12}$ Moreover, in vivo imaging and postmortem studies in patients with PD suggest that the loss of dopaminergic cell bodies in the substantia nigra lags behind the loss of nigrostriatal axon terminals. ${ }^{13,14}$ Taken together, these data suggest that the brunt of the initial pathology in PD may be at the level of the presynaptic terminals.

Recently, in vivo imaging of presynaptic terminals across the human brain has become feasible through the development of (R)-1-((3-(11C-methyl-11C)pyridin4-yl)methyl)-4-(3,4,5-trifluorophenyl)pyrrolidin-2-one $\left({ }^{11} \mathrm{C}-\mathrm{UCB}-\mathrm{J}\right)$, a positron emission tomography (PET) radioligand for the synaptic vesicle protein $2 \mathrm{~A}$ (SV2A). ${ }^{15,16}$ SV2A belongs to a family of transmembrane synaptic vesicle proteins that also includes SV2B and SV2C. ${ }^{17,18}$ The three SV2 proteins are differentially expressed in the brain. SV2A is ubiquitously present in presynaptic terminals throughout the brain, irrespective of their neurotransmitter type. ${ }^{17,18} \mathrm{SV} 2 \mathrm{~B}$, although more restricted in distribution, is also found in a wide variety of synapses. ${ }^{17,18}$ By contrast, SV2C is only present in a small subset of neurons in the brainstem, basal ganglia, and olfactory bulb. ${ }^{17}$

Here we performed ${ }^{11} \mathrm{C}$-UCB-J PET in patients with early PD to assess the magnitude and anatomical extent of presynaptic terminal loss across the brain. In addition, we performed PET with the selective dopamine transporter (DAT) radioligand [18F]-(E)$\mathrm{N}$-(3-iodoprop-2-enyl)-2 $\beta$-carbofluoroethoxy-3 $\beta$-( $4^{\prime}$ methyl-phenyl) nortropane $\left({ }^{18} \mathrm{~F}\right.$-FE-PE2I $)$, which permits measurement of presynaptic as well as somatodendritic DAT levels in dopaminergic neurons and thus allows a direct comparison of presynaptic versus somatodendritic damage within the nigrostriatal tract. ${ }^{13,19-21}$

\section{Materials and Methods}

\section{Subjects}

Patients with PD were recruited in the Movement Disorders Clinic of the University Hospitals in Leuven from October 2018 to December 2019. Healthy controls were partners of patients with PD or were recruited via advertisements in the hospital and local newspapers. Inclusion criteria for patients were clinical diagnosis of PD based on the Movement Disorder Society (MDS) clinical diagnostic criteria, ${ }^{22}$ disease duration less than 5 years since the onset of motor symptoms as reported by the patient, Hoehn and Yahr stage 1 or 2 in medication on state, and an abnormal ${ }^{123}$ I-FP-CIT Single Photon Emission Computed Tomography (SPECT) confirming clinical diagnosis. Patients with neuropsychiatric diseases other than PD and controls with abnormal findings on clinical neurological examination or important neuropsychiatric disorders were excluded. Shared exclusion criteria for patients and controls were history of brain surgery, major internal diseases (eg, cancer), history of alcohol or drug abuse, contraindications for magnetic resonance imaging (MRI), white matter lesion load Fazekas 2 or more, or other relevant MRI abnormalities.

The study was approved by the local ethics committee and performed in accordance with the World Medical Association Declaration of Helsinki, the guidelines for Good Clinical Practice, and General Data Protection Regulation. Written informed consent from every subject was obtained before the start of the study. The study was registered on ClinicalTrials.gov (NCT04243304).

\section{Clinical Assessments}

All patients and controls underwent a comprehensive clinical assessment of motor and nonmotor manifestations. Motor signs were assessed by a certified rater (A.D.) using Part III of the MDS-Unified Parkinson's Disease Rating Scale (UPDRS) ${ }^{23}$ in medication off state, that is, after overnight withdrawal of levodopa (mean $25.4 \pm 16.2$ hours after last levodopa intake) and at least 36 hours (mean $51.3 \pm 11.2$ hours) withdrawal of ropinirole, pramipexole, rasagiline, and safinamide. ${ }^{23,24}$ Hoehn and Yahr score was determined both off and on medication. All other scales were administered on medication. A description of clinical assessments can be found in the online supporting materials. Total daily levodopa equivalent dose was calculated as described. ${ }^{25}$

\section{Image Acquisition}

All participants underwent an ${ }^{18}$ F-FE-PE2I PET magnetic resonance (MR) and ${ }^{11} \mathrm{C}-\mathrm{UCB}-\mathrm{J}$ PET computed tomography (CT) scan. Both scans took place on 2 different days in varying order depending on logistical planning, on average $51 \pm 37$ days apart.

Subjects underwent a static ${ }^{11}$ C-UCB-J PET-CT scan (General Electric MI4 PET-CT, Boston, Massachusetts, USA), acquired 60 to 90 minutes postinjection. ${ }^{26} \mathrm{~A}$ mean activity of $223 \pm 51 \mathrm{MBq}$ of ${ }^{11} \mathrm{C}-\mathrm{UCB}-\mathrm{J}$ was injected. All patients were scanned in medication off state, as defined previously.

To assess DAT binding, subjects underwent a 50 to 70 minutes postinjection static ${ }^{18} \mathrm{~F}$-FE-PE2I PET-MR scan on the simultaneous General Electric Signa 3T PET-MR. We previously demonstrated that this time window is optimal for group comparisons. ${ }^{21} \mathrm{~A}$ mean activity of $107 \pm 17 \mathrm{MBq}$ 
${ }^{18}$ F-FE-PE2I was injected. ${ }^{18}$ F-FE-PE2I PET-MR in the patients with PD was performed on medication because antiparkinsonian drugs are considered not to interfere significantly with DAT imaging. ${ }^{27}$ More information on PET and MR acquisition can be found in online supporting information.

\section{Data Analysis}

Motion correction was implemented with a rigid coregistration of all frames to the first frame.

Spatial normalization to the Montreal Neurological Institute (MNI) template was performed in PMOD (version 4.0, PMOD Technologies, Zurich, Switzerland) using the template-based 3 tissue probability maps with a fixed threshold of 0.3 to only include voxels with a high probability to belong to gray matter. Automated delineation of the midbrain, pons, and medulla was done in the PMOD PNEURO tool with the Automated Anatomical Labeling (AAL) atlas, and the PMOD Parcellation tool was used to delineate the putamen and caudate nucleus. All other regions except the substantia nigra were delineated with the Hammers atlas (N30R83). ${ }^{28,29}$ An ${ }^{18}$ F-FE-PE2I DAT template was used to obtain accurate delineation of the substantia nigra according to Fazio and colleagues. ${ }^{13}$ The individual ${ }^{18}$ F-FE-PE2I images of the 20 healthy controls were normalized with a nonlinear transformation to the MNI space in the PNEURO tool of PMOD. These 20 individual images were subsequently averaged to 1 image, the ${ }^{18}$ F-FE-PE2I DAT template. In the mesencephalon, a separate volume of interest (VOI) for the left and right substantia nigra was delineated based on a set threshold of 1.68 standardized uptake value ratio (SUVR). The individual number of voxels and volume of the bilateral substantia nigra VOIs are presented in Supporting Table S1. Parametric ${ }^{11} \mathrm{C}-\mathrm{UCB}-\mathrm{J}$ nondisplaceable binding potential $\left(\mathrm{BP}_{\mathrm{ND}}\right)$ images were computed as SUVR-1 using the centrum semiovale as a white matter reference region as previously validated. ${ }^{26}$ Similarly, $\mathrm{BP}_{\mathrm{ND}}$ images for ${ }^{18} \mathrm{~F}$-FE-PE2I were calculated with the occipital cortex as the reference region. ${ }^{21}$ Information on partial volume correction (PVC) can be found in the online supporting information.

For voxel-based morphometry (VBM) analysis, the CAT12 toolbox (version r1040, University of Jena, Jena, Germany) for Matlab (version R2018b, MathWorks, Natick, MA) was used. Voxel-based analysis in SPM (version SPM12, University College London, London, UK) was done without and with a binary gray matter, basal ganglia, and substantia nigra mask.

In patients with $\mathrm{PD}$, the contralateral brain side (CONTRA) was defined as the side in the brain contralateral to the clinically most affected body side, whereas the ipsilateral brain side (IPSI) was the opposite. In the VOI-based analysis, the mean value of CONTRA and
IPSI was used for the patients with PD and compared with the mean of the left and right side of the controls. For the dorsal striatum (caudate + putamen), caudate, putamen, and substantia nigra, an asymmetry index was calculated: ([IPSI - CONTRA]/[average IPSI + CONTRA]) $\times 100 .{ }^{13}$ In analogy to the patients, for the controls the following formula was used to calculate the asymmetry index: ([RIGHT - LEFT]/[average RIGHT + LEFT]) $\times 100$.

\section{Statistical Analysis}

Statistical tests were calculated in SPSS (version 26.0, IBM, Armonk, NY) and GraphPad Prism (version 8, GraphPad Software, San Diego, CA). Data are presented as mean \pm standard deviation. The distribution of variables was tested against normality with ShapiroWilk tests. Fisher's exact test, $\chi^{2}$ test, unpaired $t$ test (with or without Welch correction), paired $t$ test, or Mann-Whitney $U$ test were used as appropriate. Statistical significance was assessed after Bonferroni correction for multiple comparisons.

Detailed information on correlation and voxel-based analysis can be found in the online supporting information.

\section{Results}

\section{Demographic and Clinical Characteristics of Study Subjects}

A total of 30 patients with early PD and 20 healthy controls were included and underwent a detailed assessment of motor and nonmotor problems. Demographic and clinical characteristics are summarized in Table 1. Patients and controls were closely matched for age and gender (Table 1). All patients with PD were treated with dopaminergic drugs.

As expected, the patients with PD had more severe motor symptoms (MDS-UPDRS Part II) and motor signs (MDS-UPDRS Part III off medication) than controls (Table 1). Patients with PD also had significantly worse scores than controls on a global nonmotor symptom scale (MDS-UPDRS Part I), on several cognitive tests (Montreal Cognitive Assessment, Symbol Digit Modalities Test, Trail Making Test Parts A and B), on scales measuring anxiety (Parkinson's Anxiety Scale), sleep problems (Scales for Outcomes in Parkinson's Disease-Sleep), and autonomic symptoms (Scales for Outcomes in Parkinson's Disease-Autonomic) and had worse olfaction (University of Pennsylvania Smell Identification Test). A total of 10 patients with PD and none of the controls screened positive on a single-question screen for rapid eye movement sleep behavior disorder (RBD) (Table 1). 
D E L V A E T A L

TABLE 1 Demographic and clinical characteristics of study subjects

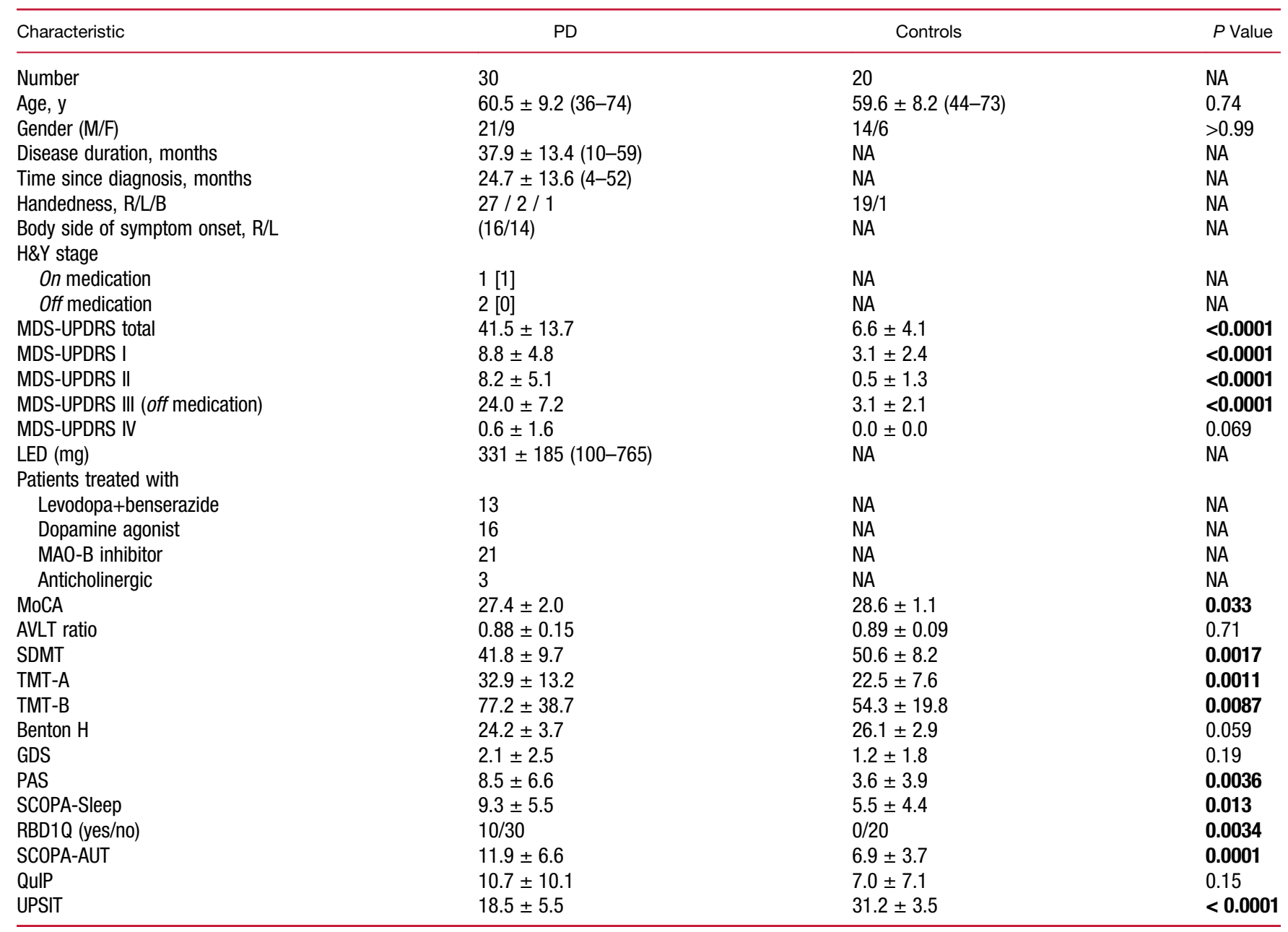

Data are given as mean $\pm \mathrm{SD}$ (range) or as median [interquartile range]. $P$ values were calculated with Fisher's exact test, $\chi^{2}$ test, unpaired $t$ tests, and Mann-Whitney $U$ test, as appropriate.

PD, Parkinson's disease; M, male; F, female; R, right; L, left; B, bimanual; H\&Y, Hoehn and Yahr stage; MDS-UPDRS, Movement Disorder Society-Unified Parkinson's Disease Rating Scale; LED, total daily levodopa equivalent dose; MAO-B, monoamine oxidase B; MoCA, Montreal Cognitive Assessment; AVLT, Auditory Verbal Learning Test, score A7/A5; SDMT, Symbol Digit Modalities Test; TMT-A, Trail Making Test Part A; TMT-B, Trail Making Test Part B; Benton H, Benton Judgment of Line Orientation test Part H; GDS, Geriatric Depression Scale; PAS, Parkinson's Anxiety Scale; SCOPA-Sleep, Scales for Outcomes in Parkinson's Disease-Sleep; SCOPA-AUT, Scales for Outcomes in Parkinson's Disease-Autonomic; RBD1Q, REM Sleep Behavior Disorder Single-Question Screen; QulP, Questionnaire for Impulsive Disorders Questionnaire; UPSIT, University of Pennsylvania Smell Identification Test; NA, not applicable.

\section{Voxel-Based Morphometry}

Age and total intracranial volume-corrected VBM revealed 2 significant clusters of decreased brain volume in patients with PD compared with controls $\left(k_{\text {extent }}=300\right.$; at threshold set peak $P_{\text {height,uncorrected }}<0.001$ and cluster level $P_{\text {Family-Wise Error (FWE) corrected }}<0.05$ ) in the left frontal and postcentral gyrus (Supporting Table S2).

\section{${ }^{11}$ C-UCB-J Group Differences}

Average ${ }^{11}$ C-UCB-J PET images for patients with PD and controls are shown in Figure 1A. VOI-based ${ }^{11} \mathrm{C}$-UCB-J $\mathrm{BP}_{\mathrm{ND}}$ group differences are shown in Figure 1B. Numerical details for all analyzed regions are shown in Supporting Table S3. In the substantia nigra, ${ }^{11} \mathrm{C}-\mathrm{UCB}-\mathrm{J} \mathrm{BP}_{\mathrm{ND}}$ was significantly lower in patients with $\mathrm{PD}$ than controls $(-15.2 \% \pm 18.2 \%$;
$P=0.002)$, even after correction for multiple comparisons (threshold $P<0.004$ for 14 regions). Patients with PD also showed a nonsignificant trend toward lower ${ }^{11} \mathrm{C}-\mathrm{UCB}-\mathrm{J}$ $\mathrm{BP}_{\mathrm{ND}}$ in the dorsal striatum $(-6.6 \% \pm 12.3 \% ; P=0.02)$, caudate $(-6.1 \% \pm 15.3 \% ; P=0.06)$, and putamen $(-5.9 \% \pm 12.5 \% ; P=0.07)$. The asymmetry index in the dorsal striatum was $0.2 \% \pm 6.9 \%$ in patients and $2.4 \% \pm 2.4 \%$ in controls $(P=0.06)$, in the caudate $0.2 \% \pm 10.0 \%$ in patients and $-1.2 \% \pm 3.8 \%$ in controls $(P=0.52)$, in the putamen $1.2 \% \pm 7.0 \%$ in patients and $-2.8 \% \pm 3.0 \%$ in controls $(P=0.009)$, and in the substantia nigra $3.4 \% \pm 15.0 \%$ in patients and $6.0 \% \pm$ $12.0 \%$ in controls $(P=0.02)$. We did not find significant group differences in the cortical regions, red nucleus, midbrain, pons, medulla oblongata, thalamus, or cerebellum. Analysis with PVC yielded similar results (Supporting Table S4 and Supporting Fig. S1). 
(a)

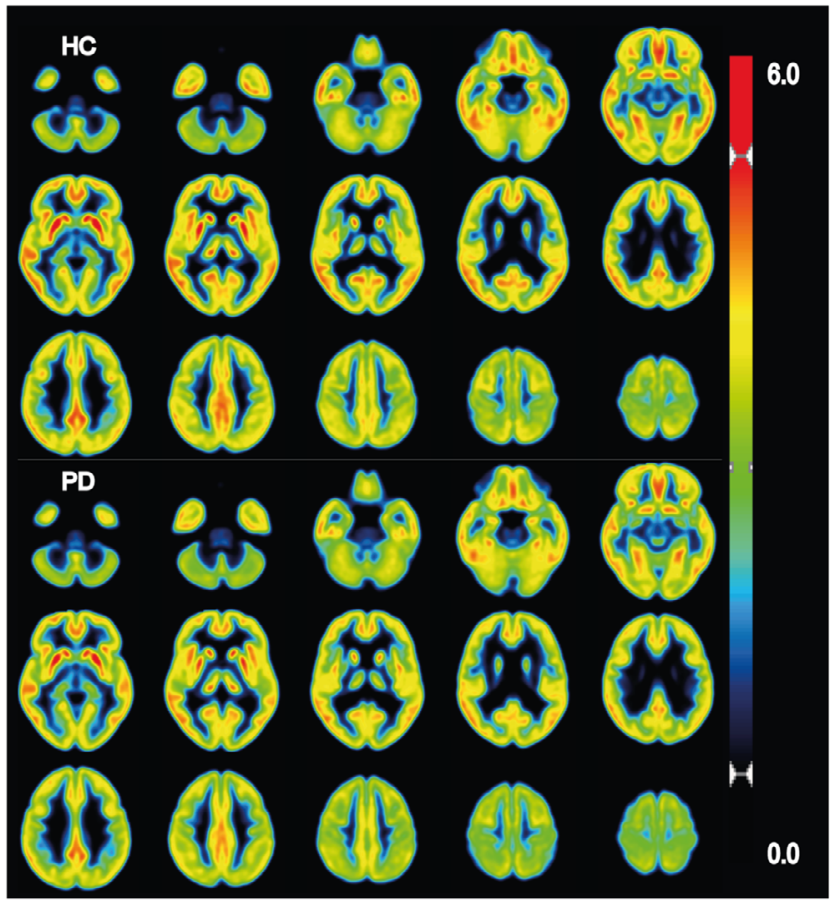

(b)

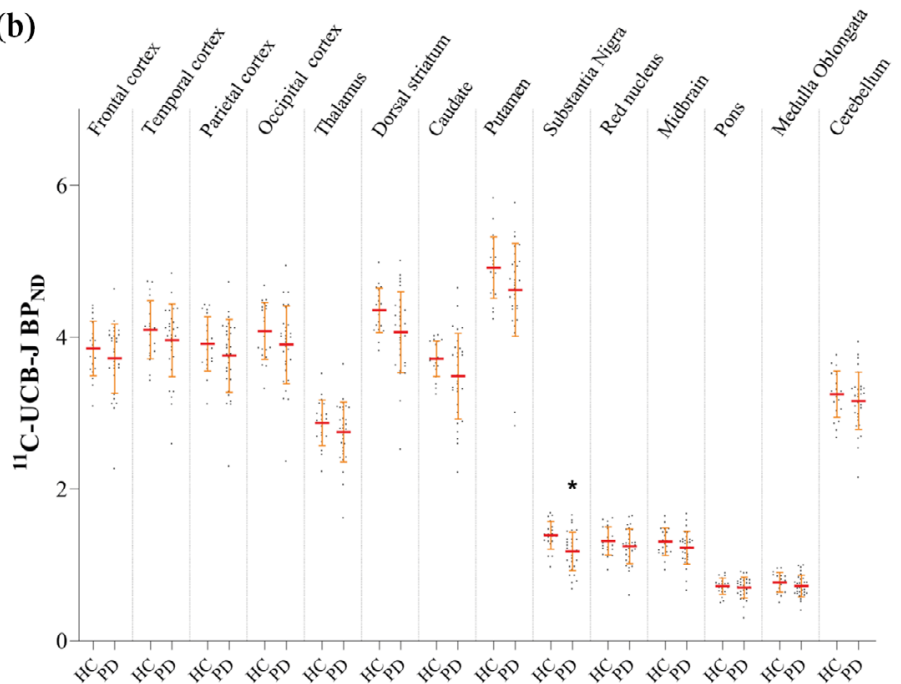

FIG $1{ }^{11} \mathrm{C}-\mathrm{UCB}-J \mathrm{BP}_{\mathrm{ND}}$ parametric images in patients with Parkinson's disease (PD) and healthy controls (HC). (A) Midtransverse average BP $\mathrm{ND}$ images of ${ }^{11}$ C-UCB-J binding in patients with PD $(n=30)$ and healthy controls $(n=20)$. Color bar represents BP the mean group ${ }^{11} \mathrm{C}-\mathrm{UCB}-J \mathrm{BP}_{\mathrm{ND}}$ values in the PD group compared with the control group $\left({ }^{*} P<0.004\right)$. Red lines show group means, standard deviations are shown in orange. No partial volume correction was applied. [Color figure can be viewed at wileyonlinelibrary.com]

Age-corrected and disease duration-corrected voxelbased analysis showed a cluster of significantly lower ${ }^{11} \mathrm{C}-\mathrm{UCB}-\mathrm{J} \mathrm{BP}_{\mathrm{ND}}$ around the putamen contralateral to the clinically most affected body side in the PD group compared with controls (Supporting Fig. S3A,C).

\section{${ }^{18}$ F-FE-PE2I Group Differences}

Average ${ }^{18}$ F-FE-PE2I PET images for patients with PD and controls are shown in Figure 2A. Results of the VOIbased analysis are summarized in Figure 2B. Patients with
PD had significant DAT loss in the dorsal striatum $(-61.5 \% \pm 11.3 \%)$, caudate nucleus $(-40.3 \% \pm 18.7 \%)$, putamen $(-75.6 \% \pm 8.1 \%)$, and substantia nigra $(-36.7 \% \pm 14.4 \%)$ with $P<0.0001$ for all regions. The asymmetry index in the dorsal striatum was $29.2 \% \pm$ $15.9 \%$ in patients and $5.5 \% \pm 5.1 \%$ in controls $(P<0.0001)$, in the caudate $19.6 \% \pm 18.1 \%$ in patients and $9.4 \% \pm 8.9 \%$ in controls $(P=0.01)$, in the putamen $43.9 \% \pm 20.1 \%$ in patients and $3.4 \% \pm 3.9 \%$ in controls $(P<0.0001)$, and in the substantia nigra $9.9 \% \pm 22.0 \%$ in patients and $-0.2 \% \pm 13.9 \%$ in controls $(P=0.06)$. 

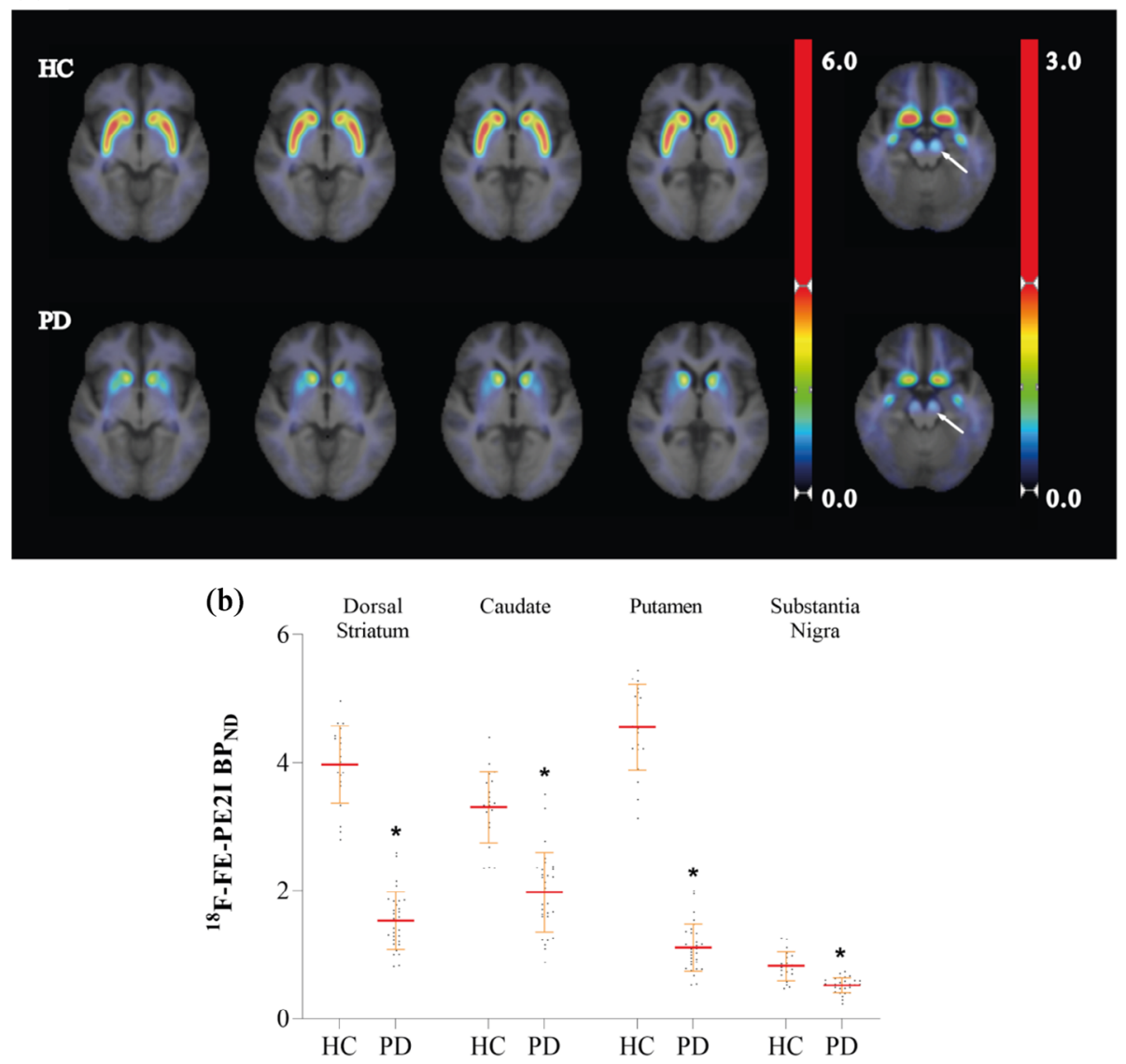

FIG $2{ }^{18} \mathrm{~F}-\mathrm{FE}-\mathrm{PE} 2 \mathrm{I} \mathrm{BP}_{\mathrm{ND}}$ images in patients with Parkinson's disease (PD) and healthy controls (HC). (A) Midtransverse average BP ${ }_{\mathrm{ND}}$ images of ${ }^{18} \mathrm{~F}-\mathrm{FE}-\mathrm{PE} 2 \mathrm{l}$ binding in patients with $\mathrm{PD}(\mathrm{n}=30)$ and $\mathrm{HC}(\mathrm{n}=20)$. White arrows indicate the substantia nigra. Color bar represents $\mathrm{BP}$ ND values. $(\mathbf{B})$ Individual and mean group ${ }^{18} \mathrm{~F}-\mathrm{FE}-\mathrm{PE} 2 \mathrm{I} \mathrm{BP}_{\mathrm{ND}}$ values in $\mathrm{PD}$ and $\mathrm{HC}$ in the dorsal striatum, caudate, putamen, and substantia nigra. Red lines show group means, standard deviations are shown in orange $\left({ }^{\star} P<0.0001\right)$. No partial volume correction was applied. [Color figure can be viewed at wileyonlinelibrary.com]

Analysis with PVC yielded similar results (Supporting Fig. S2).

Voxel-based analysis confirmed significant DAT loss in the dorsal striatum, caudate, and putamen, but not in the substantia nigra (Supporting Fig. S3B,D).

\section{Correlation of ${ }^{11} \mathrm{C}-\mathrm{UCB}-\mathrm{J}$ PET with Clinical Scores}

None of the VOIs showed a significant correlation between ${ }^{11} \mathrm{C}-\mathrm{UCB}-\mathrm{J} \mathrm{BP}_{\mathrm{ND}}$ and clinical motor or nonmotor variables. Voxel-based linear regression analysis also did not reveal any significant correlations with any of the clinical scores.

\section{Correlation of ${ }^{18}$ F-FE-PE2I PET with Clinical Scores}

VOI-based or voxel-based analysis did not reveal significant correlations of ${ }^{18}$ F-FE-PE2I $\mathrm{BP}_{\mathrm{ND}}$ values in the dorsal striatum, caudate, putamen, or substantia nigra with clinical motor or nonmotor scores.

\section{Correlation Between ${ }^{11} \mathrm{C}-U \mathrm{CB}-\mathrm{J}$ PET and ${ }^{18} \mathrm{~F}-\mathrm{FE}-\mathrm{PE} 2 \mathrm{I}$ PET}

VOI-based analysis in the PD group showed a significant positive Pearson correlation between ${ }^{11} \mathrm{C}-\mathrm{UCB}-\mathrm{J}$ binding in the caudate on the one hand and ${ }^{18} \mathrm{~F}-\mathrm{FE}-$ PE2I binding in the caudate $(r=0.63, P=0.0002)$ and the dorsal striatum $(r=0.58, P=0.001)$ on the other hand (Fig. 3A). In addition, we found a trend toward a significant correlation between ${ }^{11} \mathrm{C}-\mathrm{UCB}-\mathrm{J}$ and ${ }^{18} \mathrm{~F}-\mathrm{FE}-$ PE2I binding in the dorsal striatum $(r=0.44$, $P=0.015),{ }^{11} \mathrm{C}-\mathrm{UCB}-\mathrm{J}$ binding in the dorsal striatum and ${ }^{18}$ F-FE-PE2I binding in the caudate $(r=0.47$, $P=0.008)$, and ${ }^{11} \mathrm{C}-\mathrm{UCB}-\mathrm{J}$ binding in the substantia nigra and ${ }^{18} \mathrm{~F}-\mathrm{FE}-\mathrm{PE} 2 \mathrm{I}$ binding in the dorsal striatum $(r=0.46, P=0.012)$, caudate $(r=0.46, P=0.011)$, and substantia nigra $(r=0.43, P=0.018)$.

Homologous voxel-based Pearson correlation analysis confirmed the correlation between ${ }^{18} \mathrm{~F}-\mathrm{FE}-\mathrm{PE} 2 \mathrm{I}$ and ${ }^{11}$ C-UCB-J BP $\mathrm{ND}$ in the caudate $(r=0.40-0.99$, $P<0.05)$ and substantia nigra $(r=0.40-0.63$, $P<0.05$ ) (Fig. 3B). 

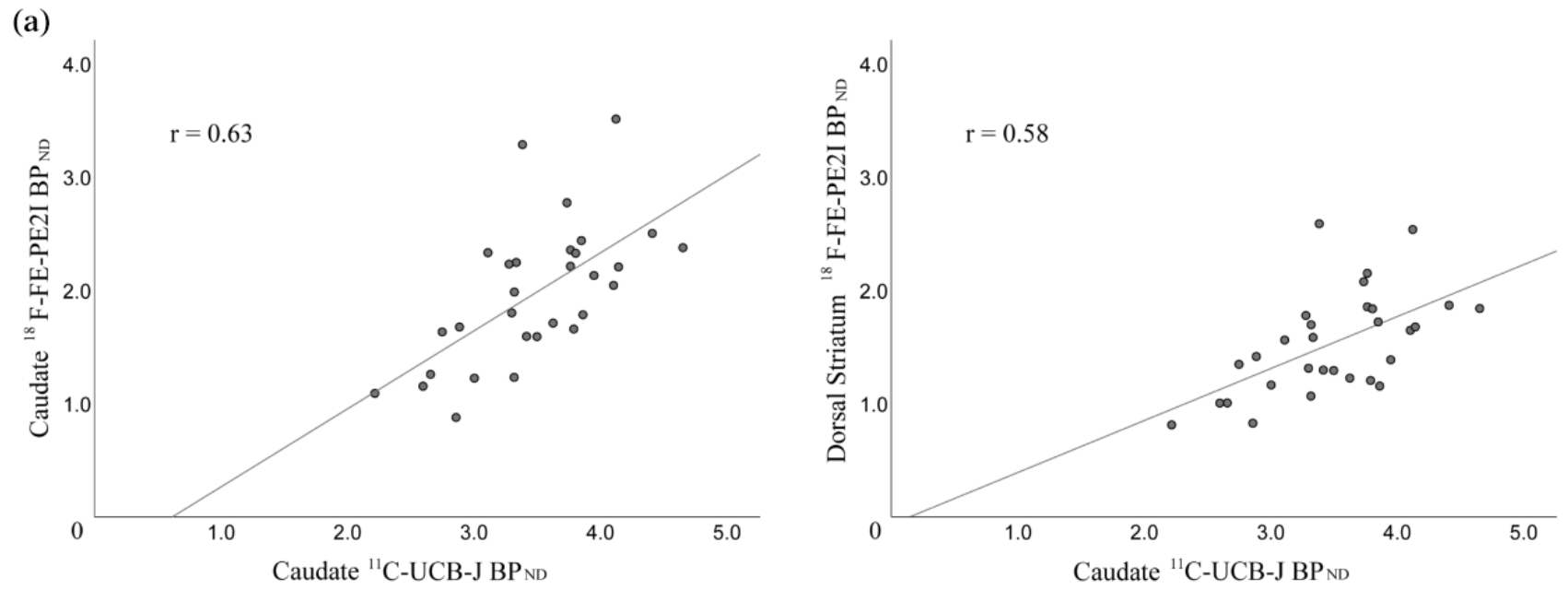

(b)

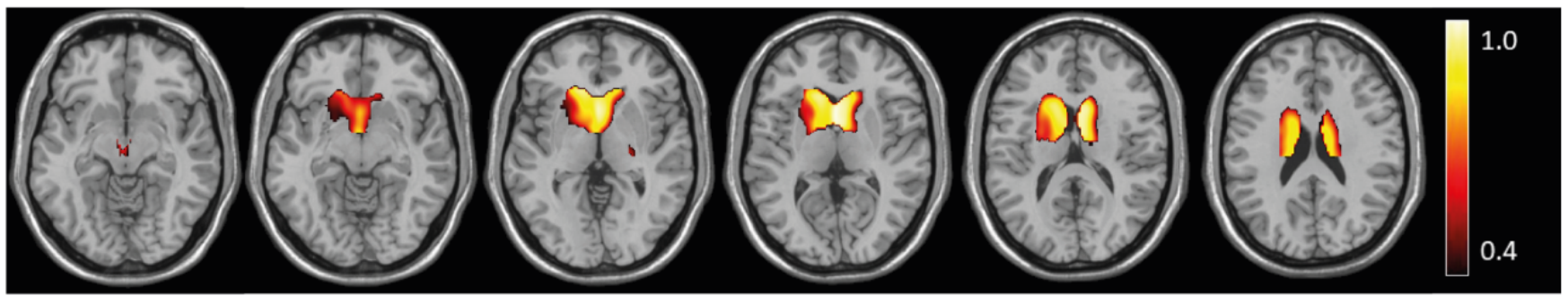

FIG 3 Correlation between ${ }^{18} \mathrm{~F}-\mathrm{FE}-\mathrm{PE} 2 \mathrm{l}$ and ${ }^{11} \mathrm{C}-\mathrm{UCB}-\mathrm{J}$ PET BP $\mathrm{ND}$ in Parkinson's disease. Data are shown without partial volume correction. (A) Graphs showing significant volume of interest-based Pearson correlations between ${ }^{11} \mathrm{C}-\mathrm{UCB}-\mathrm{J}$ and ${ }^{18} \mathrm{~F}-\mathrm{FE}-\mathrm{PE} 2 \mathrm{I}$ BP $\mathrm{ND}$. (B) Homologous voxel-based Pearson correlation analysis between ${ }^{18} \mathrm{~F}-\mathrm{FE}-\mathrm{PE} 2 \mathrm{I}$ and ${ }^{11} \mathrm{C}-\mathrm{UCB}-\mathrm{J}$ PET BP $\mathrm{ND}_{\mathrm{ND}}$. The positive correlations between ${ }^{18} \mathrm{~F}-\mathrm{FE}-\mathrm{PE} 2 \mathrm{I}$ and ${ }^{11} \mathrm{C}-\mathrm{UCB}-\mathrm{J}$ BP $\mathrm{ND}$ are shown in the caudate nucleus $(r=0.40-0.99)$ and the substantia nigra $(r=0.40-0.63)$ using $P<0.05$ and a basal ganglia and a substantia nigra mask to minimize noise $r>0.40$ and $\mathrm{K}_{\text {extent }} 20$ voxels. Color bar shows correlation coefficients. No obvious negative correlations were found. [Color figure can be viewed at wileyonlinelibrary.com]

Analysis with PVC showed comparable results, shown in Supporting Figure S4.

\section{Discussion}

PD has been hypothesized to primarily affect presynaptic terminals, ${ }^{30}$ but this hypothesis is mainly based on postmortem data ${ }^{12,14}$ and studies in cell culture and animal models. $5,6,11,31$ Here, we provide in vivo evidence for presynaptic terminal damage in patients with early PD.

SV2A is strongly concentrated in presynaptic boutons, is essentially undetectable in cell bodies, and is present in virtually all presynaptic terminals. ${ }^{15,18}$ These characteristics make SV2A an excellent marker to compare changes in density of presynaptic terminals between different brain regions. Using ${ }^{11} \mathrm{C}$-UCB-J PET to assess overall presynaptic terminal density in patients with early PD, we found significant loss of SV2A binding in the substantia nigra. We detected no significant changes in other brain areas, such as the red nucleus, which is adjacent to the substantia nigra. Thus, PD causes loss not only of nigrostriatal neurons but also of presynaptic boutons that terminate in the substantia nigra. Nigral dopaminergic neurons receive direct input from diverse brain structures including the dorsal striatum, globus pallidus, subthalamic nucleus, pedunculopontine nucleus, and amygdala. ${ }^{32,33}$ Our findings do not reveal whether these afferents are all affected to the same degree or differentially lost depending on intrinsic vulnerability determinants such as $\alpha$-synuclein expression levels. ${ }^{34}$

Several hypothetical mechanisms can be envisaged to explain the observed loss of presynaptic terminals in the substantia nigra. One possibility is that the loss of presynaptic boutons in the nigra reflects an axonal response to target deprivation. Retraction of target-deprived axon terminals after target lesioning has been described in animals, ${ }^{35,36}$ but whether similar phenomena also occur in humans with neurodegenerative diseases is not clear. Alternatively, the loss of nigral presynaptic boutons could potentially point to trans-synaptic spreading of the pathology. Studies in mice and monkeys have shown that injection of synthetic $\alpha$-synuclein fibrils or PD patient-derived Lewy body extracts into the substantia nigra or striatum induces propagation of synucleinopathy to distant brain 
regions that are anatomically connected to the inoculation site, suggesting that the pathology spreads trans-synaptically. ${ }^{5-7,37}$ Our finding of presynaptic terminal loss specifically in the substantia nigra could potentially fit with a trans-synaptic pattern of propagation of the disease process.

In addition to comparing changes in presynaptic terminal density between different brain regions with ${ }^{11} \mathrm{C}$-UCB-J PET, we used ${ }^{18} \mathrm{~F}$-FE-PE2I PET to compare presynaptic versus somatodendritic damage within the nigrostriatal tract. Nigrostriatal neurons express DAT not only on their axon terminals but also in the somatodendritic compartment. ${ }^{38}$ Unlike traditional DAT imaging techniques such as ${ }^{123}$ I-FP-CIT-SPECT, the improved spatial resolution and superior DAT selectivity of ${ }^{18}$ F-FE-PE2I PET allows to measure DAT levels both in the striatum and the substantia nigra. We found that DAT levels in patients with early PD were reduced by $61.5 \%$ in the dorsal striatum $(-75.6 \%$ in the putamen, $-40.3 \%$ in the caudate) and to a lesser extent $(-36.7 \%)$ in the substantia nigra. This is consistent with the only previous ${ }^{18}$ F-FE-PE2I PET study that compared striatal and nigral DAT levels in $\mathrm{PD}^{13}$ and also with postmortem data showing marked loss of dopaminergic markers in the striatum in early PD despite relative preservation of nigral cell bodies. ${ }^{14}$ These data strongly suggest that the presynaptic terminals of dopaminergic neurons are more affected in early PD than their cell bodies and dendrites and that pathology within these neurons may spread retrogradely from axon terminals to the somatodendritic compartment. This is reminiscent of the retrograde propagation of $\alpha$-synuclein pathology from axon to cell body that was observed in cultured neurons after addition of exogenous $\alpha$-synuclein fibrils. ${ }^{11}$

The absence of significant loss of SV2A binding in the striatum $\left(-6.6 \%\right.$ as measured by ${ }^{11} \mathrm{C}-\mathrm{UCB}-\mathrm{J}$ PET) may seem surprising in light of the profound loss of striatal DAT binding $\left(-61.5 \%\right.$ by ${ }^{18}$ F-FE-PE2I PET). Several factors may explain this paradox. First, dopaminergic terminals constitute only a minority among the total population of presynaptic terminals in the striatum. Second, although nigrostriatal neurons express SV2A, ${ }^{18}$ they also prominently express SV2C. ${ }^{17,39}$ It has been shown that each synaptic vesicle contains a relatively fixed number of SV2 molecules (five SV2 copies per vesicle). ${ }^{40}$ As a result of the presence of SV2C, the abundance of SV2A in striatal dopaminergic synaptic vesicles is probably lower than in certain other terminals, which would further diminish the sensitivity of striatal ${ }^{11} \mathrm{C}$ UCB-J binding to dopaminergic terminal loss. Third, the observed loss of DAT binding may not only be attributed to the loss of entire dopaminergic terminals but also in part to selective DAT downregulation, which may occur as a compensatory mechanism in early $\mathrm{PD}^{41}$ and could explain why striatal DAT loss is disproportionately high compared with striatal SV2A loss.
Strengths of our study were the combination of SV2A and DAT imaging, the sample sizes that were relatively large for a multimodal PET study, and the in-depth clinical phenotyping of all subjects. There were also several limitations. We only investigated markers of structural presynaptic integrity and did not assess synaptic function. Another limitation was that several small structures of interest, such as the dorsal motor nucleus of the vagus nerve and the locus coeruleus, could not be resolved and accurately quantified with the applied PET imaging instrumentation (spatial resolution of about 4-5 mm). Also, we cannot be certain whether the observed reduction of SV2A and DAT binding reflects loss of entire presynaptic boutons or a more selective loss of these proteins; however, in either case the loss of SV2A or DAT reflects a disease-related change in the presynaptic terminal. Finally, we cannot exclude the possibility that treatment with antiparkinsonian medication had an effect on SV2A binding. However, this seems unlikely because ${ }^{11} \mathrm{C}-\mathrm{UCB}-\mathrm{J}$ PET was performed after withdrawal of PD drugs and there was no correlation between SV2A binding and levodopa equivalent dose.

Recently, Matuskey and colleagues ${ }^{42}$ published the first ${ }^{11}$ C-UCB-J study in patients with PD. Similar to our results, they found that the substantia nigra showed the largest reduction in SV2A binding of all brain regions, and they confirmed lower SV2A in the substantia nigra using postmortem ${ }^{3} \mathrm{H}-\mathrm{UCB}-\mathrm{J}$ autoradiography. However, the magnitude of in vivo SV2A loss in the nigra $(-45 \%)$ was larger than in our study $(-15.2 \%)$, and they found additional significant reductions of SV2A binding in several other regions such as the red nucleus and areas in the cerebral cortex. Several factors may account for these seeming discrepancies. First, their patients were probably more advanced given the longer duration since diagnosis (4 years compared with 2 years in our study) and worse motor scores. Also, sample size was smaller (12 patients and 12 controls) than in our study, and correction for multiple comparisons was not performed. Regarding differences in imaging methods, Matuskey and colleagues ${ }^{42}$ used a high resolution research tomograph PET, which has even better spatial resolution than the state-of-the art Time-of-Flight PET-MR used in our study. Nonetheless, the fact that 2 independent ${ }^{11} \mathrm{C}$-UCB-J PET studies found that the loss of SV2A binding was greatest in the substantia nigra despite differences in study design and imaging methods strongly corroborates this finding.

We did not identify significant correlations of ${ }^{18} \mathrm{~F}-\mathrm{FE}-$ PE2I or ${ }^{11} \mathrm{C}$-UCB-J BP $\mathrm{ND}_{\mathrm{ND}}$ with clinical variables. The ${ }^{18}$ F-FE-PE2I PET study by Fazio and colleagues ${ }^{13}$ also detected no correlation between ${ }^{18}$ F-FE-PE2I BP ${ }_{\mathrm{ND}}$ and disease duration and motor scores, whereas $\mathrm{Li}$ and colleagues ${ }^{43}$ reported significant negative correlations of ${ }^{18}$ F-FE-PE2I $\mathrm{BP}_{\mathrm{ND}}$ with motor severity. In the 
${ }^{11}$ C-UCB-J PET study by Matuskey and colleagues, ${ }^{42}$ SV2A $\mathrm{BP}_{\mathrm{ND}}$ was also not correlated with clinical variables. In our study, we recruited patients with early-stage PD because we were mainly interested in the pathogenic mechanisms underlying the early phases of PD. As a result, our cohort was clinically rather homogeneous, which may have made it more difficult to detect correlations of the imaging markers with clinical scores. Longitudinal ${ }^{11} \mathrm{C}-\mathrm{UCB}-\mathrm{J}$ PET studies will be required to determine whether the rate of decline in regional presynaptic terminal density in patients with PD correlates with clinical progression in the motor and nonmotor domains.

Based on ${ }^{18}$ F-FE-PE2I PET imaging, we can conclude that axon terminals appear to be the most vulnerable part of nigrostriatal dopamine neurons in patients with early PD. On the other hand, ${ }^{11} \mathrm{C}$-UCB-J suggests that overall loss of presynaptic terminals is most prominent in the substantia nigra, which could potentially be consistent with a trans-synaptic route of spread of the disease process. Longitudinal SV2A imaging studies may shed new light on the patterns of anatomical progression of PD and their relationship with motor and nonmotor deterioration.

Acknowledgments: The authors are grateful to Mr. Kwinten Porters and Mr. Jef Van Loock for their contribution to the scanning and data handling, the positron emission tomography radiopharmacy team, and the medical physics team of University Hospitals Leuven for their skilled contributions.

\section{References}

1. Poewe W, Seppi K, Tanner CM, et al. Parkinson disease. Nat Rev Dis Primers 2017;3:17013.

2. Hirsch EC, Orieux G, Muriel MP, Francois C, Feger J. Nondopaminergic neurons in Parkinson's disease. Adv Neurol 2003;91:29-37.

3. Braak H, Del Tredici K, Rub U, de Vos RA, Jansen Steur EN, Braak E. Staging of brain pathology related to sporadic Parkinson's disease. Neurobiol Aging 2003;24(2):197-211.

4. Del Tredici K, Braak H. Review: sporadic Parkinson's disease: development and distribution of alpha-synuclein pathology. Neuropathol Appl Neurobiol 2016;42(1):33-50.

5. Recasens A, Dehay B, Bove J, et al. Lewy body extracts from Parkinson disease brains trigger alpha-synuclein pathology and neurodegeneration in mice and monkeys. Ann Neurol 2014;75(3):351-362.

6. Luk KC, Kehm V, Carroll J, et al. Pathological alpha-synuclein transmission initiates Parkinson-like neurodegeneration in nontransgenic mice. Science 2012;338(6109):949-953.

7. Masuda-Suzukake M, Nonaka T, Hosokawa M, et al. Pathological alpha-synuclein propagates through neural networks. Acta Neuropathol Commun 2014;2:88.

8. Jakes R, Spillantini MG, Goedert M. Identification of two distinct synucleins from human brain. FEBS Lett 1994;345(1):27-32.

9. Kramer ML, Schulz-Schaeffer WJ. Presynaptic alpha-synuclein aggregates, not Lewy bodies, cause neurodegeneration in dementia with Lewy bodies. J Neurosci 2007;27(6):1405-1410.

10. Schulz-Schaeffer WJ. The synaptic pathology of alpha-synuclein aggregation in dementia with Lewy bodies, Parkinson's disease and Parkinson's disease dementia. Acta Neuropathol 2010;120(2):131-143.
11. Volpicelli-Daley LA, Luk KC, Patel TP, et al. Exogenous alphasynuclein fibrils induce Lewy body pathology leading to synaptic dysfunction and neuron death. Neuron 2011;72(1):57-71.

12. Orimo S, Uchihara T, Nakamura A, et al. Axonal alpha-synuclein aggregates herald centripetal degeneration of cardiac sympathetic nerve in Parkinson's disease. Brain 2008;131(Pt 3):642-650.

13. Fazio P, Svenningsson P, Cselenyi Z, Halldin C, Farde L, Varrone A. Nigrostriatal dopamine transporter availability in early Parkinson's disease. Mov Disord 2018;33(4):592-599.

14. Kordower JH, Olanow CW, Dodiya HB, et al. Disease duration and the integrity of the nigrostriatal system in Parkinson's disease. Brain 2013;136(Pt 8):2419-2431.

15. Finnema SJ, Nabulsi NB, Eid T, et al. Imaging synaptic density in the living human brain. Sci Transl Med 2016;8(348):348ra396.

16. Heurling K, Ashton NJ, Leuzy A, et al. Synaptic vesicle protein 2A as a potential biomarker in synaptopathies. Mol Cell Neurosci 2019;97:34-42.

17. Janz R, Südhof TC. SV2C is a synaptic vesicle protein with an unusually restricted localization: anatomy of a synaptic vesicle protein family. Neuroscience 1999;94(4):1279-1290.

18. Bajjalieh SM, Frantz GD, Weimann JM, McConnell SK, Scheller RH. Differential expression of synaptic vesicle protein 2 (SV2) isoforms. J Neurosci 1994;14(9):5223-5235.

19. Sasaki T, Ito H, Kimura Y, et al. Quantification of dopamine transporter in human brain using PET with 18F-FE-PE2I. J Nucl Med 2012;53(7):1065-1073.

20. Jakobson Mo S, Axelsson J, Jonasson L, et al. Dopamine transporter imaging with [(18)F]FE-PE2I PET and [(123)I]FP-CIT SPECT-a clinical comparison. EJNMMI Res 2018;8(1):100.

21. Delva A, Van Weehaeghe D, van Aalst J, et al. Quantification and discriminative power of (18)F-FE-PE2I PET in patients with Parkinson's disease. Eur J Nucl Med Mol Imaging 2020;47(8): 1913-1926.

22. Postuma RB, Berg D, Stern M, et al. MDS clinical diagnostic criteria for Parkinson's disease. Mov Disord 2015;30(12):1591-1601.

23. Goetz CG, Tilley BC, Shaftman SR, et al. Movement Disorder Society-sponsored revision of the Unified Parkinson's Disease Rating Scale (MDS-UPDRS): scale presentation and clinimetric testing results. Mov Disord 2008;23(15):2129-2170.

24. Athauda D, Maclagan K, Skene SS, et al. Exenatide once weekly versus placebo in Parkinson's disease: a randomised, double-blind, placebo-controlled trial. Lancet 2017;390(10103):1664-1675.

25. Tomlinson CL, Stowe R, Patel S, Rick C, Gray R, Clarke CE. Systematic review of levodopa dose equivalency reporting in Parkinson's disease. Mov Disord 2010;25(15):2649-2653.

26. Koole M, van Aalst J, Devrome M, et al. Quantifying SV2A density and drug occupancy in the human brain using [(11)C]UCB-J PET imaging and subcortical white matter as reference tissue. Eur J Nucl Med Mol Imaging 2019;46(2):396-406.

27. Morbelli S, Esposito G, Arbizu J, et al. EANM practice guideline/SNMMI procedure standard for dopaminergic imaging in parkinsonian syndromes 1.0. Eur J Nucl Med Mol Imaging 2020;47 (8):1885-1912.

28. Hammers A, Allom R, Koepp MJ, et al. Three-dimensional maximum probability atlas of the human brain, with particular reference to the temporal lobe. Hum Brain Mapp 2003;19(4):224-247.

29. Tzourio-Mazoyer N, Landeau B, Papathanassiou D, et al. Automated anatomical labeling of activations in SPM using a macroscopic anatomical parcellation of the MNI MRI single-subject brain. Neuroimage 2002;15(1):273-289.

30. Wong YC, Luk K, Purtell K, et al. Neuronal vulnerability in Parkinson disease: should the focus be on axons and synaptic terminals? Mov Disord 2019;34(10):1406-1422.

31. Lundblad M, Decressac M, Mattsson B, Bjorklund A. Impaired neurotransmission caused by overexpression of alpha-synuclein in nigral dopamine neurons. Proc Natl Acad Sci U S A 2012;109(9): 3213-3219.

32. Lee CR, Tepper JM. Basal ganglia control of substantia nigra dopaminergic neurons. J Neural Transm Suppl 2009(73):71-90. 
33. Watabe-Uchida M, Zhu L, Ogawa SK, Vamanrao A, Uchida N. Whole-brain mapping of direct inputs to midbrain dopamine neurons. Neuron 2012;74(5):858-873.

34. Henderson MX, Cornblath EJ, Darwich A, et al. Spread of alphasynuclein pathology through the brain connectome is modulated by selective vulnerability and predicted by network analysis. Nat Neurosci 2019;22(8):1248-1257.

35. Marty S, Weinitz JM, Peschanski M. Target dependence of adult neurons: pattern of terminal arborizations. J Neurosci 1994;14(9): 5257-5266.

36. Rossi F, Jankovski A, Sotelo C. Target neuron controls the integrity of afferent axon phenotype: a study on the Purkinje cell-climbing fiber system in cerebellar mutant mice. J Neurosci 1995;15(3 Pt 1): 2040-2056.

37. Goedert M, Masuda-Suzukake M, Falcon B. Like prions: the propagation of aggregated tau and alpha-synuclein in neurodegeneration. Brain 2017;140(2):266-278.

38. Hersch SM, Yi H, Heilman CJ, Edwards RH, Levey AI. Subcellular localization and molecular topology of the dopamine transporter in the striatum and substantia nigra. J Comp Neurol 1997;388(2): 211-227.

39. Dunn AR, Stout KA, Ozawa M, et al. Synaptic vesicle glycoprotein 2C (SV2C) modulates dopamine release and is disrupted in
Parkinson disease. Proc Natl Acad Sci U S A 2017;114(11): E2253-E2262.

40. Mutch SA, Kensel-Hammes P, Gadd JC, et al. Protein quantification at the single vesicle level reveals that a subset of synaptic vesicle proteins are trafficked with high precision. J Neurosci 2011;31(4):1461-1470.

41. Nandhagopal R, Kuramoto L, Schulzer M, et al. Longitudinal evolution of compensatory changes in striatal dopamine processing in Parkinson's disease. Brain 2011;134(Pt 11):3290-3298.

42. Matuskey D, Tinaz S, Wilcox KC, et al. Synaptic changes in Parkinson's disease assessed with in-vivo imaging. Ann Neurol 2020;87(3):329-338.

43. Li W, Lao-Kaim NP, Roussakis AA, et al. (11) C-PE2I and (18) F-Dopa PET for assessing progression rate in Parkinson's: a longitudinal study. Mov Disord 2018;33(1):117-127.

\section{Supporting Data}

Additional Supporting Information may be found in the online version of this article at the publisher's web-site. 


\section{SGML and CITI Use Only DO NOT PRINT}

\section{Author Roles}

(1) Research project: A. Conception, B. Organization, C. Execution; (2) Statistical Analysis: A. Design, B. Execution, C. Review and Critique; (3) Manuscript: A. Writing of the first draft, B. Review and Critique.

A.D.: $1 \mathrm{~B}, 1 \mathrm{C}, 2 \mathrm{~A}, 2 \mathrm{~B}, 2 \mathrm{C}, 3 \mathrm{~A}, 3 \mathrm{~B}$

D.V.W.: 2B, 2C, 3B

M.K.: 1B, 2A, 2C, 3B

K.V.L.: 1A, 1B, 1C, 2A, 2C, 3B

W.V.: 1A, 1B, 1C, 2A, 2C, 3A, 3B

\section{Full financial disclosures for the previous 12 months}

Aline Delva and Donatienne Van Weehaeghe are PhD fellows of the Fund for Scientific Research Flanders. Wim Vandenberghe is a Senior Clinical Investigator of the Fund for Scientific Research Flanders. This study was supported by KU Leuven ("Opening the Future" and C24/17/063) and UZ Leuven (Fund for Academic Studies and Klinische Onderzoeks-en Opleidingsraad). There are no other financial disclosures or conflicts of interest. 